\title{
Optical system design and integration of the Mercury Laser Altimeter
}

Luis Ramos-izquierdo ${ }^{1}$, V. Stanley Scott $\mathrm{III}^{2}$, Stephen Schmidt ${ }^{3}$, Jamie Britt ${ }^{4}$, William Mamakos ${ }^{5}$, Raymond Trunzo ${ }^{6}$, John Cavanaugh ${ }^{7}$, Roger Miller $^{8}$

The Mercury Laser Altimeter (MLA), developed for the 2004 MESSENGER mission to Mercury, is designed to measure the planet's topography via laser ranging. A description of the MLA optical system and its measured optical performance during instrument-level and spacecraft-level integration and testing are presented.

\section{Introduction}

The Mercury Laser Altimeter (MLA) is one of seven scientific instruments on board the MErcury Surface, Space ENvironment, GEochemistry, and Ranging (MESSENGER) spacecraft, the first orbiter mission to the planet Mercury. MESSENGER is scheduled to launch in August 2004 and arrive at Mercury in March 2011 for a one-year (four Mercury years) study. The MLA laser time-of-flight measurement together with the spacecraft orbit positional data will help determine the planet's surface elevation, libration, and internal structure [1-3]. MLA was designed and developed at NASA's Goddard Space Flight Center (GSFC) over a period of two and a half years and delivered to The Johns Hopkins University Applied Physics Laboratory (JHU/APL) on June 30, 2003, for spacecraft-level integration. This paper describes the optical system and the optical integration and testing of MLA. 


\section{Instrument Description}

MLA's top-level optical specifications are listed in Table 1. The transmitter and receiver specifications are based on experience with earlier space-based laser altimeters, such as the Mars Orbiter Laser Altimeter (MOLA) and the Geoscience Laser Altimeter System (GLAS), but modified for the MLA ranging geometry, the low planet albedo, and the high infrared (IR) flux and Solar background of Mercury [4]. MLA has to perform range measurements from a distance of up to $800 \mathrm{~km}$, at a slant angle of up to $53^{\circ}$, and in daytime during part of the mission, all of which drove the laser energy, the laser divergence, and the receiver telescope aperture requirements. The laser repetition rate was constrained by available instrument power. The receiver telescope field-of-view (FOV) specification was a compromise: the FOV is narrow enough to make solar background noise negligible during most of the MESSENGER orbits and wide enough to allow for a reasonable instrument boresite alignment margin.

An assembly drawing of MLA is shown in Figure 1. The MLA structure is made of optical-grade beryllium for its low mass, high stiffness, and high thermal capacitance. The beryllium components were designed in-house and fabricated by Axsys Technologies. The Main Housing holds the electronic sub-assemblies and serves as an optical bench for the Laser Transmitter and the four Receiver Telescopes. The Laser Transmitter [5] is built on a small beryllium bench that mounts to the Main Housing center compartment. An external $15 \mathrm{X}$ beam expander telescope mounted to the laser bench sets the final transmitted laser beam divergence. A Reference Cube attached to the Main Housing is used to monitor the laser pointing angle during MLA integration and environmental testing and to transfer the MiLA iaser alignment information to the 
spacecraft coordinate system. The four MLA Receiver Telescopes are mounted on the corners of the Main Housing. The output signal from each telescope is fiber-coupled to the Detector/Aft-Optics assembly mounted underneath the Main Housing. The Detector/Aft-Optics assembly combines, filters, and re-images the output from all four fiber optics onto a single SiAPD detector. The fiber optics are all the same length for return pulse timing reasons and are routed and secured along delin channels attached to the outer edge of the Main Housing underside. MLA is mounted to the MESSENGER composite instrument deck via three titanium flexures, and the structure is fully enclosed by thermal blankets such that only the five optical apertures remain open to the external environment.

The main factors that drove the opto-mechanical design of MLA were the tight constraints on instrument mass $(7.3 \mathrm{~kg})$, volume $(300 \times 300 \times 300 \mathrm{~mm})$, and peak power (23 $\mathrm{W})$ and the challenging mission thermal environment. MESSENGER will be in a 12-hour, highly eccentric elliptical orbit around Mercury [6] with only a 30-45 minute MLA science measurement period over the planet's northern hemisphere where the surface temperature can range from $110^{\circ} \mathrm{K}$ on the planet's dark side to over $700^{\circ} \mathrm{K}$ on the sub-Solar region [7]. During the balance of the orbit, MLA cools off by radiating heat to deep space. MLA will be "thermal cycled" in this fashion over 700 times during the course of the MESSENGER mission. Figure 2 shows the predicted instrument temperatures at the beginning and end of the operational science phase for the MESSENGER $280^{\circ}$ true anomaly orbit (TA280), a noon-midnight orbit close to the MESSENGER "hot" case. The MLA optical system is required to operate over a wide temperature range, in a non-steady-state, and with large thermal gradients. The 
instrument design constraints and mission thermal environment required an integrated optical, mechanical, and thermal instrument design.

The MLA optical alignment requirements are listed in Table 2 and are divided into integration, alignment, and stability requirements. The MLA opto-mechanical design philosophy was to minimize the number of sub-assembly and instrument level adjustments required to align the instrument in order to better ensure alignment stability. The optical and mechanical components were toleranced such that upon initial instrument integration the boresite error between the laser and the four receiver telescopes was less than the \pm 2 mrad receiver telescope line-of-sight adjustment range. Laser pointing knowledge and stability relative to the MESSENGER instrument deck are key alignment parameters since this information is used to determine the laser footprint location on the planet surface. Once the instrument was integrated and the boresite alignment completed, the pointing angle stability of the transmitted laser beam and the boresite alignment of the Receiver Telescopes were measured and tracked during the MLA and MESSENGER environmental test programs.

\section{Receiver Telescope}

The four MLA Receiver Telescopes have a combined aperture of $417 \mathrm{~cm}^{2}$ and a $400 \mu \mathrm{rad}$ diameter nominal FOV. The collecting area is equivalent to a single $0.25-\mathrm{m}$ diameter telescope with a $15 \%$ secondary and spider obscuration factor. The original MLA receiver concept was based on a scaled-down version of the beryllium Cassegrain telescopes used on MOLA (0.5-m diameter) and GLAS (1.0-m diameter), but once the MESSENGER thermal environment was better understood it became apparent that this 
telescope would not meet the MLA on-orbit performance requirements. Although the MOLA and GLAS telescopes are athermal (to $1^{\text {st }}$ order) under a bulk temperature change, they are very sensitive to axial and radial thermal gradients due to the high coefficient of thermal expansion (CTE) of beryllium and the large longitudinai magnification and fast primary of the Cassegrain telescope design [8]. The multi-aperture, refractive MLA Receiver Telescope design is not athermal, but this optical design can handle thermal gradients an order of magnitude larger than an equivalent beryllium Cassegrain telescope for a comparable amount of image degradation. The MLA Receiver telescope operating thermal range is $20^{\circ} \mathrm{C} \pm 25^{\circ} \mathrm{C}$ and the survival thermal range is $-30^{\circ} \mathrm{C}$ to $+60^{\circ} \mathrm{C}$.

The optical layout of the MLA Receiver Telescope is shown in Figure 3. The telescope is a four-element reverse telephoto design with a $500-\mathrm{mm}$ focal length, a 300-mm unfolded path length, and a final speed of $f / 4.35$. The plano-convex objective lens is made of sapphire and has a focal length of $230 \mathrm{~mm}$, a diameter of $125 \mathrm{~mm}$, and a mounted clear aperture diameter of $115 \mathrm{~mm}$. Sapphire was selected for all the optics exposed to the Mercury environment for its ability to withstand thermal shocks [9], its lower absorption in the IR compared to optical glasses, and its resistance to radiation darkening. Although sapphire is birefringent and can generate double images, its imaging performance is adequate for the MLA receiver "photon bucket". Ten high-purity, synthetic sapphire blanks were manufactured by Crystal Systems and the blanks ground and polished into lenses by Meller Optics. A negative focal length triplet lens group increases the focal length of the objective lens and corrects spherical aberration and coma. The triplet was manufactured out of radiation-resistant Schott BK7G18 by Optimax Systems. The telescope is folded in order to fit within the ailocated MíA 
volume, which also helped reduce the cantilevered mass. The dielectric fold mirror only reflects a small spectral band centered at $1064 \mathrm{~nm}$, which provides protection against an accidental view of the Sun since most of the visible solar radiation will go through the fold mirror and scatter off its frosted backside onto the MESSENGER instrument deck.

A picture of one of MLA Receiver Telescopes is shown in Figure 4. Each of the four telescopes is identical except for the clocking orientation of the section folded underneath the Main Housing. The telescope tube, the mirror mount, and the fiber mount are made of optical-grade beryllium. The lenses are clamped in place with titanium flexures and the pre-load adjusted by machining the thickness of an internal spacer. The clearance between the lenses and the tube bore is only $25 \mu \mathrm{m}$ on the radius in order to minimize vibration-induced boresite shifts. The fold mirror is bonded into place with space-qualified GE RTV566. The optical and mechanical components were toleranced such that upon initial integration, the telescope optical axis was perpendicular to its mounting flange to within $1 \mathrm{mrad}$. The only Receiver Telescope optical adjustment is at the fiber-optic connector where a shim can be adjusted to set focus and the connector decentered on oversized mounting holes to adjust the telescope line-of-sight over a $\pm 2 \mathrm{mrad}$ range. Each Receiver Telescope assembly weighs $740 \mathrm{~g}$, driven mostly by the 400-g sapphire objective.

A $200-\mu \mathrm{m}$ core diameter, $0.22 \mathrm{NA}$, multimode, step-index fiber-optic assembly at the focal plane of each telescope yields the $400-\mu$ rad diameter FOV. The fiber-optic assemblies are similar to the ones flown on GLAS, and were fabricated in-house at Goddard's Advanced Photonic Interconnection Manufacturing Laboratory (Code 562). Key requirements for the fiber-optic assemblies were that the fibers be well centered $( \pm$ 
$10 \mu \mathrm{m})$ on their connectors and that the fiber connector interface be repeatable in both focus $( \pm 10 \mu \mathrm{m})$ and de-center $( \pm 5 \mu \mathrm{m})$ in order to allow for replacement of the fiber optics without the need to re-focus or re-boresite the MLA Receiver Telescopes. Diamond AVIMS connectors were selected for this application because they provide a keyed, repeatable, and rugged interface. All the fiber ends were anti-reflection (AR) coated at $1064 \mathrm{~nm}$ by Denton Vacuum to increase their average transmission to $97 \%$. $300-\mu \mathrm{m}$ core diameter fiber optics providing a $600-\mu \mathrm{rad}$ diameter FOV were also fabricated and tested in case instrument-level environmental testing indicated that we needed the additional boresite alignment margin. The Receiver Telescope and the Detector/Aft-Optics assembly were designed to operate with either fiber size. The completed fiber-optic assemblies were tested for vacuum, temperature, vibration, and radiation effects [10] prior to instrument integration.

As mentioned earlier the MLA Receiver Telescope design is not athermal. Optothermal analysis using both paraxial equations and optical design software (Zemax) showed that the refractive telescope design could tolerate a $\pm 30^{\circ} \mathrm{C}$ bulk temperature change before its blur circle diameter increased to $\sim 100 \mu \mathrm{m}$ or $\sim 200 \mu \mathrm{rad}$. The net effect of the thermal de-focus is that the telescope nominal top-hat FOV becomes trapezoidal (Figure 5); all FOV plots have the same 400- $\mu$ rad full-width-at-half-maximum (FWHM) but the FOV size above $90 \%$ normalized transmission is only half as wide with the telescope at $+50^{\circ} \mathrm{C}$ (or at $-10^{\circ} \mathrm{C}$ ) as it is at the nominal telescope alignment temperature of $20^{\circ} \mathrm{C}$. The main reason for the telescope thermal de-focus is the large and positive $\mathrm{dn} / \mathrm{dT}$ (change in index of refraction with temperature) of sapphire which makes the telescope focal length shrink as the telescope tube expands with increasing temperature 
and vice versa. We could not find a suitable set of optical and mechanical materials that could athermalize the telescope while still meeting all the other MLA Receiver Telescope design requirements. The opto-thermal performance of the MLA Receiver Telescope is adequate, but it does reduce our boresite alignment margin during the hot MESSENGER noon-midnight orbits.

During the noon-midnight orbits the IR flux from Mercury into each Receiver Telescope aperture can be up $40 \mathrm{~W}$. The sapphire objective lens will absorb $\sim 50 \%$ of this IR flux and transmit the balance to the telescope tube. Since MLA is not nadir-pointing during these orbits the inside of the telescope tubes will not be symmetrically illuminated. We used a combination of optical and thermal computer-aided-design (CAD) programs to model the telescope thermal profile during the TA240 orbit, another noon-midnight orbit close to the MESSENGER "hot" case. The goal of the opto-thermal analysis was to calculate the thermally induced receiver boresite shift due to the asymmetric telescope tube illumination. Custom software interfaces were developed by Lambda Research Inc. (TracePro, Optical Software for Layout and Optimization or OSLO) and Harvard Thermal Inc. (Thermal Analysis System or TAS) to transfer information between the codes. The Receiver Telescope opto-thermal model accounted for both changes to the objective lens shape and index of refraction and mechanical deformations of the beryllium telescope tube. The thermal analysis was performed by Harvard Thermal Inc. based on the calculated absorbed IR flux from the TracePro Mercury-MLA model. The thermal analysis showed that the Receiver Telescope would develop the expected $\sim 30^{\circ} \mathrm{C}$ axial gradient plus a $\sim 10^{\circ} \mathrm{C}$ radial gradient (Figure 6). The "perturbed" optical system was then raytraced with OSLO to calculate the effects on image size and location: the 
image de-focused as expected, but the telescope line-of-sight change was only $15 \mu \mathrm{rad}$, which is small enough to neglect.

We fabricated five aluminum engineering model (EM) Receiver Telescopes, an aluminum EM Main Housing, and a set of EM fiber-optic assemblies in order to develop the receiver integration procedures and test setups, determine the optimum routing for the fiber-optic assemblies, and troubleshoot any hardware interference issues. The EM Receiver Telescope test program included characterizing the lens mounting flexures and calculating the required thicknesses of the internal spacers to obtain the correct lens mounting pre-loads, installing and focusing the fiber-optic assemblies including compensating for operation in vacuum, measuring the telescope image quality (blur circle) and FOV, measuring the telescope optical axis relative to its mounting flange, measuring the telescope line-of-sight shift under different orientations to gravity, performing survival and operational thermal tests, measuring the boresite effects of radial thermal gradients on the telescope tube, and measuring the stray light characteristics of the completed assembly. Figure 7 shows the change in the Receiver Telescope 200- $\mu \mathrm{m}$ fiber optic back-illuminated image and the receiver telescope $400-\mu \mathrm{rad}$ FOV between air (1 ATM) and vacuum ( 0 ATM) operation. The test results at 0 ATM validated our calculation of the shim thickness required for vacuum operation (we first focus the system in air and then adjust the shim thickness per our calculated change in telescope back-focal distance with pressure). The size and shape of the FOV plot at the in-focus 0 ATM setting also indicated that the telescope imaging performance was adequate and that the as-fabricated focal length was correct. The testing of the EM Receiver Telescopes validated our optical, mechanical, and thermal models and indicated that the 
increased stiffness and lower CTE provided by the beryllium flight hardware were indeed required to meet the MLA alignment stability and opto-thermal performance goals.

All optical substrates and coatings were space-qualified, and the opto-mechanical parts inspected and precision cleaned prior to the flight integration of the MLA optical assemblies. All optical materials, including the bandpass filter and the fiber-optic assemblies, were tested before and after exposure to $50 \mathrm{krad}$ of total ionizing gamma radiation with no measurable difference in transmission at $1064 \mathrm{~nm}$. The sapphire optics were AR coated with a proprietary double layer AR coating from Quality Thin Films, and coated witness samples were thermal cycled 100 times between $-20^{\circ} \mathrm{C}$ and $+70^{\circ} \mathrm{C}$ per MIL-C-48497 and tested for adhesion and severe abrasion resistance per MIL-C-675-C with no measurable degradation. AR-coated witness samples for the laser beam expander optics were also tested and qualified for laser damage threshold level by Spica Inc. The completed MLA flight optical assemblies were space-qualified per GSFC's General Environmental Verification Specification (GEVS) guidelines prior to instrument-level integration and the flight integration process documented per GSFC's ISO-9001 guidelines. Table 3 is a summary of the thermal qualification of the MLA optical assemblies and their component sub-assemblies. A total of six flight Receiver Telescopes, twenty flight Fiber-Optic assemblies, two flight Aft-Optics assemblies, and two flight Laser Transmitter Telescopes were integrated, tested, and delivered to the MLA instrument integration and test (I\&T) team. 


\section{Aft-Optics Assembly}

The MLA Aft-Optics assembly collimates the output of each Receiver Telescope fiberoptic, combines the four beams such that they go through a common bandpass filter, and re-images all four fibers onto a single spot on a $0.7-\mathrm{mm}$ diameter SiAPD detector (Figure 8). We had previous experience with the detector (MOLA, GLAS) and with the narrow bandpass filter (GLAS), but fiber-coupling the telescope to the detector was a new approach for us. The main optical challenge was achieving a design that allowed for coupling multiple fiber-optics onto a single detector; the design also had to be compact to fit in the allocated space under the Main Housing.

The collimating lenses are 11-mm focal length Geltech aspheres with $2 \%$ cerium added to the Corning C0550 substrate material to prevent radiation darkening. The two imaging lenses are made from radiation-resistant Schott SF6G05 and have a combined focal length of $18.6 \mathrm{~mm}$. The optical system images the input fiber-optics at a $1.7 \mathrm{X}$ magnification to yield a detector-illuminated spot size $0.34 \mathrm{~mm}$ in diameter. The collimated beams have a divergence of $\pm 9 \mathrm{mrad}$ which is within the acceptance angle of the bandpass filter, a $0.7-\mathrm{nm}$ FWHM, two cavity, temperature-stabilized interference filter from Barr Associates [11] with a peak transmission of $88 \%$ at $1064.4 \mathrm{~nm}$. The angle of incidence (AOI) of the collimated beams on the bandpass filter can be adjusted by up to $3^{\circ}$ off-normal by de-centering the Aft-Optics fiber-optic connectors in order to peak the transmission at the MLA laser wavelength $(1064.3 \mathrm{~nm})$. A test fixture allowed for coupling a portion of the MLA EM laser beam into the Aft-Optics assembly fiber-optics while simultaneously monitoring the transmitted laser energy and the location of the fiber images on the assembly focal plane in order to ensure that all four channels were 
wavelength-tuned to the MLA laser and imaged into a common spot. The Aft-Optics can be aligned in air and operated in vacuum without any vacuum de-focus compensation since the change in collimation and imaging with pressure is negligible. Thermal defocus and beam de-collimation over the thermal operating range is aiso small and can be neglected.

The Aft-Optics assembly is $\sim 75 \times 50 \times 50 \mathrm{~mm}$, weighs 204 grams, has an operational thermal range of $20^{\circ} \mathrm{C} \pm 20^{\circ} \mathrm{C}$, and a survival thermal range of $-30^{\circ} \mathrm{C}$ to $+40^{\circ} \mathrm{C}$. The mechanical components are titanium to match the CTE of the optics. The BK7G18 fold prism and mirror are bonded with Scotch-Weld 2216 Grey epoxy and the rest of the optics are held with retainer rings. The Aft-Optics assembly mates to the beryllium detector bench via an interface plate that allows for focus and de-center adjustment. The Aft-Optics assembly is aligned to the MLA detector by looking through one of the fiber-optic connector ports with a small charge-coupled-device (CCD) camera while the other three fibers illuminate the focal plane. The interface plate thickness is adjusted until the detector image is in focus and the whole Aft-Optics assembly is decentered until the three illuminating spots are centered on the detector (Figure 9). After the detector alignment is completed, the Aft-Optics fiber connectors and interface plate are "liquid pinned" with Scotch-Weld 2216 Grey epoxy. The mated Detector/Aft-Optics assembly (Figure 10) is then installed on the Main Housing and electrically integrated to the detector power supply and signal processing electronics. 


\section{Laser Transmitter Telescope}

The Laser Transmitter Telescope is a $15 \mathrm{X}$ magnification, afocal beam expander with a 45-mm diameter output clear aperture. The magnification was derived from the

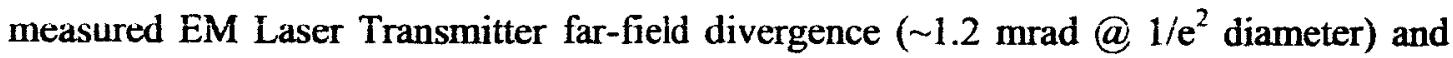
the required final transmitted laser beam divergence $\left(80 \mu \mathrm{rad} @ 1 / \mathrm{e}^{2}\right.$ diameter); the output clear aperture was established by the beam expander magnification and the input laser beam size $(\sim 2 \times 2 \mathrm{~mm})$. The MLA Laser Transmitter Telescope is designed to operate over a $20^{\circ} \mathrm{C} \pm 40^{\circ} \mathrm{C}$ temperature range without any significant increase in the transmitted laser beam divergence and to survive over a $-30^{\circ} \mathrm{C}$ to $+75^{\circ} \mathrm{C}$ temperature range. The Laser Transmitter Telescope assembly (Figure 11) is $180 \mathrm{~mm}$ long, $56 \mathrm{~mm}$ diameter (max.), and weighs $180 \mathrm{~g}$.

The beam expander is a Galilean optical design with a Corning 7980 fused silica negative lens, a BK7G18 positive lens group, and a sapphire exit window (Figure 12). The sapphire window adds thermal-shock protection by adding thermal mass and reducing the IR flux directly absorbed by the positive lens group. A non-sequential raytrace analysis insured that no beam expander ghost beams were focused on any of the beam expander or laser train optical surfaces. The mechanical design is similar to that of the Receiver Telescopes: the beam expander tube is optical-grade beryllium, a titanium flexure is used to mount the positive lens group and the sapphire window, and the clearance between the lenses and the tube bore is only $25 \mu \mathrm{m}$ on the radius. The negative lens is mounted in a small titanium cell with an internal shim to allow for focus adjustment. The optical and mechanical components were toleranced to achieve an optical axis-to-mechanical mounting flange error of $<1 \mathrm{mrad}$ in order to meet our 
instrument-level integration requirements. The beam expander dominates the pointing of the transmitted laser beam since any laser angular input errors are reduced by a factor of 15 while a tilt of the Laser Transmitter Telescope assembly leads to an almost 1:1 change [1:14/15 exactly] in the laser beam pointing angle.

The MLA beam expander design is not athermal, but its performance over temperature is more than adequate. BK7G18 has a much smaller $d n / d T$ coefficient than sapphire, and the change in focal length of the positive lens group with temperature partially compensates for the change in beryllium tube length. An opto-thermal analysis performed with the CODE $\mathrm{V}$ diffraction-based beam propagation module predicts a farfield divergence of $<60 \mu \mathrm{rad}$ over the $20^{\circ} \mathrm{C} \pm 40^{\circ} \mathrm{C}$ operational thermal range. (The nominal divergence at $20^{\circ} \mathrm{C}$ is only $50 \mu$ rad because CODE $\mathrm{V}$ assumes an ideal $\mathrm{M}^{2}=1.0$ TEM00 input beam.) Since the sapphire window/BK7G18 lens combination at the top of the beam expander absorbs most of the IR flux from Mercury there is no asymmetric illumination of the beam expander tube that might lead to a line-of-sight change as was the case with the Receiver Telescopes.

We fabricated two aluminum EM Laser Transmitter Telescopes in order to validate our optical, mechanical, and thermal models, develop the beam expander integration procedure and test setups, and perform assembly-level testing with the EM Laser Transmitter. The Laser Transmitter Telescope bolts to the laser bench, and the laser beam is aligned to the beam expander with a set of Risley prisms (to adjust beam angle) and tilt plates (to adjust beam de-center). After integration of the EM beam expander to the EM Laser Transmitter the laser team discovered that feedback from the beam expander de-stabilized the laser oscillator. The laser team increased the AOI on the 
beam expander to $\sim 7.5 \mathrm{mrad}$ and added a polarizer and $1 / 4$ waveplate to the laser optical train to add optical isolation between the beam expander and the laser oscillator. After these two changes were implemented the EM Laser Transmitter assembly performance became nominal again.

The most difficult part of the beam expander integration process was setting focus for $1064 \mathrm{~nm}, 0$-ATM operation. A tolerance analysis showed that we needed to adjust the negative lens spacing to an accuracy of about $25 \mu \mathrm{m}$. To achieve this accuracy we first focused the beam expander for plane-wavefront output at $633 \mathrm{~nm}, 1$ ATM using a Zygo interferometer. Because the distance between the laser beam waist and the beam expander input optic is much less than the laser Rayleigh range, the Gaussian focal shift is very small and can be ignored. For all practical purposes, the beam expander focal setting that yields the lowest far-field divergence is the afocal setting. The interferometer allowed for precise collimation at $633 \mathrm{~nm}, 1$ ATM; we then adjusted the beam expander lens spacing in several steps to obtain collimation at $1064 \mathrm{~nm}, 0$ ATM. The beam expander collimation procedure is described below.

The beam expander was setup in a double pass configuration with the positive lens group facing the interferometer and the flat surface of the negative lens acting as the reference mirror. By observing the transmitted wavefront amplitude and curvature (convex vs. concave) and reproducing the observed wavefront error in CODE V we were able to quickly converge on the required shim thickness for 633-nm, 1-ATM collimation. We then measured the beam expander performance with a continuous wave $(\mathrm{CW}) \mathrm{HeNe}$ laser to confirm the focal setting established with the Zygo interferometer. The next step was to adjust the shim thickness for 1064-nm, 1-ATM operation using the glass melt 
index-of-refraction data for the positive group lenses. We then confirmed the beam expander new focal setting with a CW 1064-nm laser. One final shim thickness adjustment was made to re-focus the beam expander for 0-ATM operation using a pressure de-focus number calculated both paraxially and with Zemax. The completed Laser Transmitter Telescope was then placed in a vacuum chamber and its performance at 0 ATM verified with the CW $1064-\mathrm{nm}$ laser. The ratio of the size of the laser far-field images with and without the beam expander in the path verified that the assembly was correctly focused for 1064-nm, 0-ATM operation (Figure 13).

Two flight model (FM) Laser Transmitter Telescopes were integrated, tested, and delivered to the laser team. In addition to functional testing and thermal qualification, we also performed a vibration qualification test to verify the alignment stability of the mounted Laser Transmitter Telescope. We measured the beam expander optical axis relative to the laser bench reference mirror before and after the vibe test. To measure the beam expander optical axis, the assembly was placed between two theodolites with one theodolite aligned to the laser bench reference mirror and the other one serving as a surrogate laser beam (Figure 14). The "transmitter" theodolite focus was adjusted to compensate for the beam expander residual optical power at 1 ATM such that its image on the "reference" theodolite was in focus and de-magnified by 15X. (Care must be taken that the beam expander assembly transverse position in the test setup be very repeatable since the MLA FM beam expander is not afocal at 1 ATM.) The spare FM Laser Transmitter Telescope assembly underwent 60 seconds of random vibration to a 6.8 Grms level about all three axes. No measurable motion was observed between the beam expander optical axis and the iaser bench reference mirror. 


\section{MLA Optical Integration and Testing}

The MLA instrument subsystems were sequentially integrated into the Main Housing: first the mounting flexures and the Reference Cube were installed, then the electronic sub-assemblies and electrical harnesses were integrated and tested; this was followed by the integration and testing of the Laser Transmitter and the Detector/Aft-Optics assemblies, and finally the Receiver Telescopes were attached and the fiber-optic assemblies connected, routed, and secured. MLA was then installed on the alignment ground support equipment (GSE) plate at which point the instrument was ready for boresite alignment and instrument-level functional testing. A picture of MLA fully integrated and mounted on the alignment GSE plate is shown in Figure 15.

Two key pieces of equipment were developed to boresite MLA: a collimator system and a laser beam dump. The main collimator system optic is a $2.5-\mathrm{m}$ focal length, 400-mm diameter Space Optics Research Labs (SORL) off-axis parabola (OAP). A 50:50 beamsplitter cube placed near the focal plane of the OAP generates two focal planes: one focal plane has a target reticule and a $C C D$ camera, while the other focal plane has a 1064-nm single-mode (SM) fiber-optic source mounted on a computer controlled XY stage. A second CCD camera looks at the two focal planes through the fourth optical surface of the beamsplitter cube to verify that the target reticule and the SM fiber source are coincident and in focus. The purpose of the laser beam dump is to attenuate the MLA laser output beam without changing its pointing angle or far-field divergence. The laser beam dump reflects $90 \%$ of the MLA laser energy into a diffuser/lens/fiber assembly used to monitor the MLA laser energy. The transmitted MLA laser beam is further âttenuàted using Schơti KG glasss absorption fiters. The laser beam dump pick-off 
beamsplitter and attenuation filters were custom made to have wedge angles $<5 \mu \mathrm{rad}$ each. KG glass transmits in the visible so the laser beam dump assembly transmitted wavefront and beam deviation error can be measured with an interferometer or a pair of theodolites. The laser beam dump assembly met our line-of-sight deviation error goal of $<10 \mu \mathrm{rad}$ after all the required attenuation filters were installed.

The MLA boresite alignment techniques and test set-ups were derived from those developed for MOLA [12]. To boresite the instrument the MLAVGSE plate assembly is installed on the collimator instrument stand with MLA looking down. Having the gravity axis parallel to the instrument optical axis minimizes any gravity effects on the MLA transmitter and receiver lines of sight. The MLA boresite procedure is straightforward: first the MLA laser is attenuated with the laser beam dump and its output directed to the center of the collimator target reticule, then the receiver telescope fiber optics are backilluminated at $1064 \mathrm{~nm}$ and the fiber-optic connectors de-centered until the four fiberoptic images are centered on the collimator target reticule (Figure 16). (The laser and receiver images are out of focus because the instrument alignment is performed at 1 ATM while all the optical assemblies are focused for 0 ATM.) After the boresite alignment procedure is completed the receiver telescope fiber-optic connectors are "liquid pinned" with Scotch-Weld 2216 Grey epoxy. To verify the MLA boresite alignment, the FOV of each receiver telescope is measured by moving the collimator SM fiber-optic source in two orthogonal axes while recording the output of the MLA detector. Symmetric, wellcentered FOV cross-sectional profiles would indicate that the MLA receiver telescopes are properly boresited to the MLA laser. The shape and size of all the FOV profiles were as expected for $1 \mathrm{ATM}$, and all were within our $\pm 50 \mu \mathrm{rad}$ boresite alignment requirement. 
The combined cross-sectional FOV of all four MLA receiver telescopes is shown in Figure 17.

Once the boresite alignment was completed the MLA instrument underwent environmental qualification. The MLA instrument vibration test levels were $8.0 \mathrm{Grms}$ about the $X$ and $Y$ axes and $9.9 \mathrm{Grms}$ about the $\mathrm{Z}$ axis, the instrument optical axis; the full level random vibes lasted 60 seconds per axis. We measured the following MLA alignment parameters before and after the vibe test: (1) the pointing of the MLA laser relative to the MLA Reference Cube, (2) the alignment of the MLA Reference Cube relative to a reference cube bonded to the alignment GSE plate, and (3) the boresite alignment of the four MLA Receiver Telescopes. We found no motion $(<10 \mu \mathrm{rad})$ between the MLA laser and the MLA Reference Cube and a small amount of motion $(\sim 50 \mu \mathrm{rad})$ between the MLA instrument and the alignment GSE plate. All the Receiver Telescopes moved relative to the MLA Laser, but only telescope S/N 3 (T3) was significantly out of its boresite alignment allocation after the vibration qualification test (Figure 18). Although we found a small electronic cable lodged between the RMU/CPU Housing and the back end of Receiver Telescope $S / \mathrm{N} 3$, re-routing the cable did not bring the alignment back. Further troubleshooting of the $\mathrm{S} / \mathrm{N} 3$ receiver telescope proved inconclusive, so we decided to proceed with instrument-level thermal vacuum (TVAC) testing before taking any action regarding the boresite alignment of the $\mathrm{S} / \mathrm{N} 3$ receiver telescope.

The MLA TVAC test lasted several weeks and included both hot and cold cycles that encompassed MLA's survival and operational thermal ranges. The MLA TVAC test configuration is shown in Figure 19. MLA is mounted on an Invar plate that simulates 
the MESSENGER low-CTE composite instrument deck. An aluminum frame holds the Invar plate, a thermal target plate that simulates the IR heat load from Mercury, and an optical target assembly. The optical target assembly is blanketed and temperature controlled to insure its optical stability. A small tube goes from the MLA Laser Transmitter Telescope to the optical target assembly to enclose the MLA laser beam and prevent any scatter from saturating the very sensitive MLA detector during operational tests. The optical target assembly performs several functions: a fiber-coupled diffuser source is used to inject test signals into one of the Receiver Telescopes (S/N 1) to test the MLA detector and ranging electronics, the laser beam dump is used to monitor the MLA laser energy and attenuate the transmitted laser beam, and a lateral transfer retro-reflector (LTR) and motorized set of Risley prisms flip the attenuated MLA laser beam back into one of the receiver telescopes ( $\mathrm{S} / \mathrm{N} 4)$ to measure its FOV profile. In addition, a small collimator/CCD camera system mounted outside the TVAC chamber is used to monitor the pointing angle of the MLA laser relative to a reference cube mounted on the TVAC fixture Invar plate.

The MLA laser pointing angle and divergence were very stable during the course of the TVAC test. No MLA laser motions larger than $\sim 50 \mu \mathrm{rad}$ were observed during the test, even without correcting for motions and vibrations of the external collimator or the TVAC chamber. FOV cross-sectional plots of Receiver Telescope S/N 4 were generated during several hot and cold operating plateaus. This was done by plotting the MLA detector received pulse width as a function of the MLA laser beam deviation angle introduced by the optical target Risley prisms. (The detector pulse width is directly correiated to the detector incident energy aithough the reiationship is not linear.) The 
full-width of the $\mathrm{S} / \mathrm{N} 4$ receiver telescope FOV cross-sections at 0 ATM was $\sim 400 \mu \mathrm{rad}$, but the FOV edges were not as sharp as previously measured at the sub-assembly level because the MLA laser is not a point source. All the FOV plots were well centered on the MLA laser optical axis except for one trace that showed a $50 \mu \mathrm{rad}$ offset.

After the MLA TVAC test was completed we re-measured the instrument optical alignment. We found no measurable angular offset $(<10 \mu \mathrm{rad})$ between the MLA iaser and the MLA Reference Cube and only small changes $(<50 \mu \mathrm{rad})$ in the pre-TVAC boresite alignment of the four Receiver Telescopes (Figure 20). Receiver Telescope S/N 3 was still out of its boresite alignment allocation so we debated whether to re-align the telescope or increase the size of its fiber-optic assembly from $200 \mu \mathrm{m}(400 \mu \mathrm{rad})$ to 300 $\mu \mathrm{m}(600 \mu \mathrm{rad})$ to regain boresite alignment margin. Swapping the fiber-optic assembly was an easier operation but the larger fiber-optic would lead to $\sim 30 \%$ higher Solar background noise. We opted to re-align the S/N 3 telescope and to continue monitoring its boresite alignment during MESSENGER lcvel environmental testing. The MESSENGER vibration test levels and expected launch loads are lower than the MLA vibration test levels, so we felt the risk was small that the $S / N 3$ telescope would move significantly again. The final step of the MLA optical alignment procedure involved measuring and documenting the angular alignment of the MLA laser relative to the two side faces of the MLA Reference Cube that are used to transfer the MLA laser pointing angle information to the MESSENGER spacecraft coordinate system. 


\section{MLA Integration to MESSENGER}

MLA was delivered to the JHU/APL MESSENGER spacecraft I\&T team on June 30 , 2003, and integrated unto the spacecraft on July 22, 2003. An Image of MLA installed on the MESSENGER instrument deck is shown in Figure 21; the image was taken after integration of the MESSENGER instrument-deck thermal blankets. Our tolerancing of the MLA opto-mechanical components proved successful in that the MLA laser beam was found to be aligned to the spacecraft coordinate system within $0.25 \mathrm{mrad}$ and in compliance with our co-boresite alignment requirement to the Mercury Dual Imaging System (MDIS) instrument.

We continued to monitor the alignment and health of MLA during the course of the MESSENGER spacecraft environmental qualification program. The optical target assembly we used during the MLA instrument TVAC test was re-configured for MLA spacecraft-level testing. The optical target assembly allowed us to monitor the following parameters: (1) the response of the MLA detector and signal processing electronics to

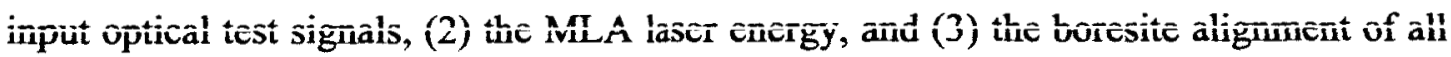
four MLA Receiver Telescopes to the MLA laser. In particular, we re-measured the MLA boresite alignment after the MESSENGER spacecraft underwent vibration testing (Figure 22) and after the spacecraft completed TVAC testing and arrived at the Astrotech facilities in Titusville, FL, for launch preparations (Figure 23). Although both the spacecraft-level vibration and TVAC tests led to MLA boresite alignment shifts, all four MLA Receiver Telescopes are still within their boresite alignment allocation for launch. All other MLA optical and electronic performance parameters remained nominal during the course of the MESSENGER envirouniental test program. 


\section{Conclusion}

MLA instrument-level integration and testing was completed on June 30, 2003, and MESSENGER spacecraft-level integration and environmental testing was completed on February 26, 2004. The MESSENGER spacecraft was successfully launched from Launch Pad 17B at Cape Canaveral Air Force Station, Fla., on August 3, 2004 aboard a three-stage Boeing Delta II rocket. As of this writing, the MESSENGER spacecraft is on its way towards the planet Mercury where it is expected to arrive and enter orbit in March 2011 .

The work presented in this paper would not have been possible without the contributions of many other individuals at NASA/GSFC. In particular we would like to thank Mr. Peter Dogoda, Mr. Sid Johnson, Mr. Ryan Simmons, Mr. Craig Stevens, Ms. Melanie Ott, Ms. Patricia Friedberg, Mr. Marcellus Proctor, Mr. Jeffrey Guzek, Dr. Danny Krebs, Mr. Randy Hedgeland, Ms. Linda Miner, Mr. Kevin W. Redman, and Mr. Jon Vermillion; our fearless leaders: Dr. Xiaoli Sun, Mr. James C. Smith, Mr. Edward

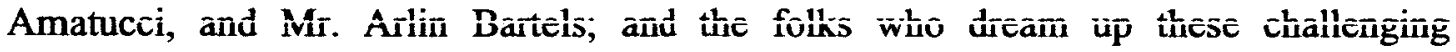
interplanetary scientific missions (and get the funding so the engineers can play): Dr. David E. Smith and Dr. Maria T. Zuber.

The authors are all contractor and civil servant engineers at NASA's Goddard Space Flight Center in Greenbelt, Maryland: ${ }^{1}$ LRI Optical Design, ${ }^{2}$ NASA/GSFC Laser Remote Sensing Branch, ${ }^{3}$ Sigma Space Corporation, ${ }^{4}$ NASA/GSFC Electro-Mechanical Systems Branch, ${ }^{5}$ Design Interface Inc., ${ }^{6}$ Swales Aerospace, ${ }^{7}$ NASA/GSFC Laser \& Electro-Optics Brañùn, ${ }^{8}$ NASA'GSFC Electrical Systems Bränch 


\section{References:}

1. S. C. Solomon, R. L. McNutt, Jr., R. E. Gold, M. H. Acuña, D. N. Baker, W. V. Boynton, C. R. Chapman, A. F. Cheng, G. Gloeckler, J. W. Head, III, S. M. Krimigis, W. E. McClintock, S. L. Murchie, S. J. Peale, R. J. Phillips, M. S. Robinson, J. A. Slavin, D. E. Smith, R. G. Strom, J. I. Trombka, and M. T. Zuber, "The MESSENGER mission to Mercury: Scientific objectives and implementation", Planetary and Space Science 49, 1445-1465, 2001.

2. R. E. Gold, S. C. Solomon, R. L. McNutt, Jr., A. G. Santo, J. B. Abshire, M. H. Acuña, R. S. Afzal, B. J. Anderson, G. B. Andrews, P. D. Bedini, J. Cain, A. F. Cheng, L. G. Evans, W. C. Feldman, R. B. Follas, G. Gloeckler, J. O. Goldsten, S. E. Hawkins, III, N. R. Izenberg, S. E. Jaskulek, E. A. Ketchum, M. R. Lankton, D. A. Lohr, B. H. Mauk, W. E. McClintock, S. L. Murchie, C. E. Schlemm, II, D. E. Smith, R. D. Starr, and T. H. Zurbuchen, "The MESSENGER mission to Mercury: Scientific payload", Planetary and Space Science 49, 1467-1479, 2001.

3. A. G. Santo, R. E. Gold, R. L. McNutt, Jr., S. C. Solomon, C. J. Ercol, R. W. Farquhar, T. J. Hartka, J. E. Jenkins, J. V. McAdams, L. E. Mosher, D. F. Persons, D. A. Artis, R. S. Bokulic, R. F. Conde, G. Dakermanji, M. E. Goss, Jr., D. R. Haley, K. J. Heeres, R. H. Maurer, R. C. Moore, E. H. Rodberg, T. G. Stern, S. R. Wiley, B. G. Williams, C. L. Yen and M. R. Peterson, "The MESSENGER mission to Mercury: Spacecraft and mission design", Planetary and Space Science 49, 1481-1500, 2001. 
4. X. Sun, J. F. Cavanaugh, J. C. Smith, and A. E. Bartels, "Design and performance measurement of the Mercury Laser Altimeter", 2004 Conference on Lasers and Electro-Optics (CLEO), CThN3 presentation, May 2004.

5. D. J. Krebs, A. M. Novo-Gradac, and S. X. Li, "Compact, passively Q-switched Nd:YAG laser for the MESSENGER mission to the planet Mercury", to be published in Applied Optics.

6. C. J. Ercol and A. G. Santo, "Determination of optimum phase angles at Mercury perihelion for an orbiting spacecraft", Society of Automotive Engineers (SAE) International Conference on Environmental Systems, Denver, CO, Session: Thermal and Environmental Control Simulation Software II - Applications Journal, 1999-01-2123, July 1999.

7. J. F. Clawson, "Thermal environments", Document JPL D-8160, Jet Propulsion Laboratory, California Institute of Technology, January 1991.

8. P. Generie and W. Hayden, "Estimation of the on-orbit distortion of the Mars Orbiter Laser Altimeter (MOLA II) primary mirror", Advanced Materials for Optical and Precision Structures, Proc. Society of Photo-Optical Instrumentation Engineers (SPIE) Vol. 2857, p. 45-56, November 1996.

9. F. Schmid, C.P. Khattak, and D.M. Felt, "Sapphire sparkles in many optical elements", Laser Focus World, June 1996.

10. M. N. Ott, M. Proctor, M. Dodson, S. Macmurphy, and P. Friedberg, “Optical fiber cable assembly characterization for the Mercury Laser Altimeter", Society of Photo-Optical Instrumentation Engineers (SPIE) AeroSense Conference on 
Enabling Photonic Technologies for Aerospace Applications V, Proceedings Vol. 5104, April 2003.

11. J. R. Potter and J. C. Simons, "Stability of IAD refractory oxide narrowband interference filter", Proc. Society of Photo-Optical Instrumentation Engineers (SPIE) Vol. 1952, p. 186-191, Nov 1993.

12. L. Ramos-Izquierdo, J. L. Bufton, and P. Hayes, "Optical system design and integration of the Mars Observer Laser Altimeter", Applied Optics, Vol. 33, No. 3, p. 307-322, 20 January 1994. 
Figure Captions:

1. MLA Assembly Drawing: a) Top View, b) Bottom View

2. MLA Thermal Model Predictions for TA280 orbit:

a) Beginning of Science Phase, b) End of Science Phase

3. MLA Receiver Telescope Optical Layout

4. MLA Receiver Telescope Assembly

5. MLA Receiver Telescope Analysis: FOV vs. Temperature

6. MLA Receiver Telescope On-Orbit Temperature (TA240, End of Science)

7. MLA Receiver Telescope Vacuum De-focus Test Results

a) 1-ATM fiber image, b) 0-ATM fiber image, c) 1-ATM FOV, d) 0-ATM FOV

8. MLA Detector/Aft-Optics Optical Layout

9. MLA Detector Illumination Image

10. MLA Detector/Aft-Optics Assembly

11. MLA Laser Transmitter Telescope Assembly

12. MLA Laser Transmitter Telescope Optical Layout

13. MLA Laser Transmitter Telescope Far-Field Images at 0-ATM, 1064-nm

a) CW Laser only, b) CW Laser with MLA 15X Beam Expander

14. MLA Laser Transmitter Telescope Vibration Test Measurement Set-up

15. MLA Installed on Alignment GSE Plate

16. MLA Boresite Alignment: a) Laser Image, b) Receiver Telescope Images (4)

17. MLA Combined Receiver Telescope FOV Cross-Sections

18. MLA Boresite Alignment: Pre- and Post- Instrument Vibration Test

19. MLA TVAC Test Configuration

20. MLA Boresite Alignment: Pre- and Post- Instrument TVAC Test

21. MLA installed on MESSENGER Instrument Deck 
22. MLA Boresite Alignment: Pre- and Post- Spacecraft Vibration Test

23. MLA Boresite Alignment: Pre- and Post- Spacecraft TVAC Test 

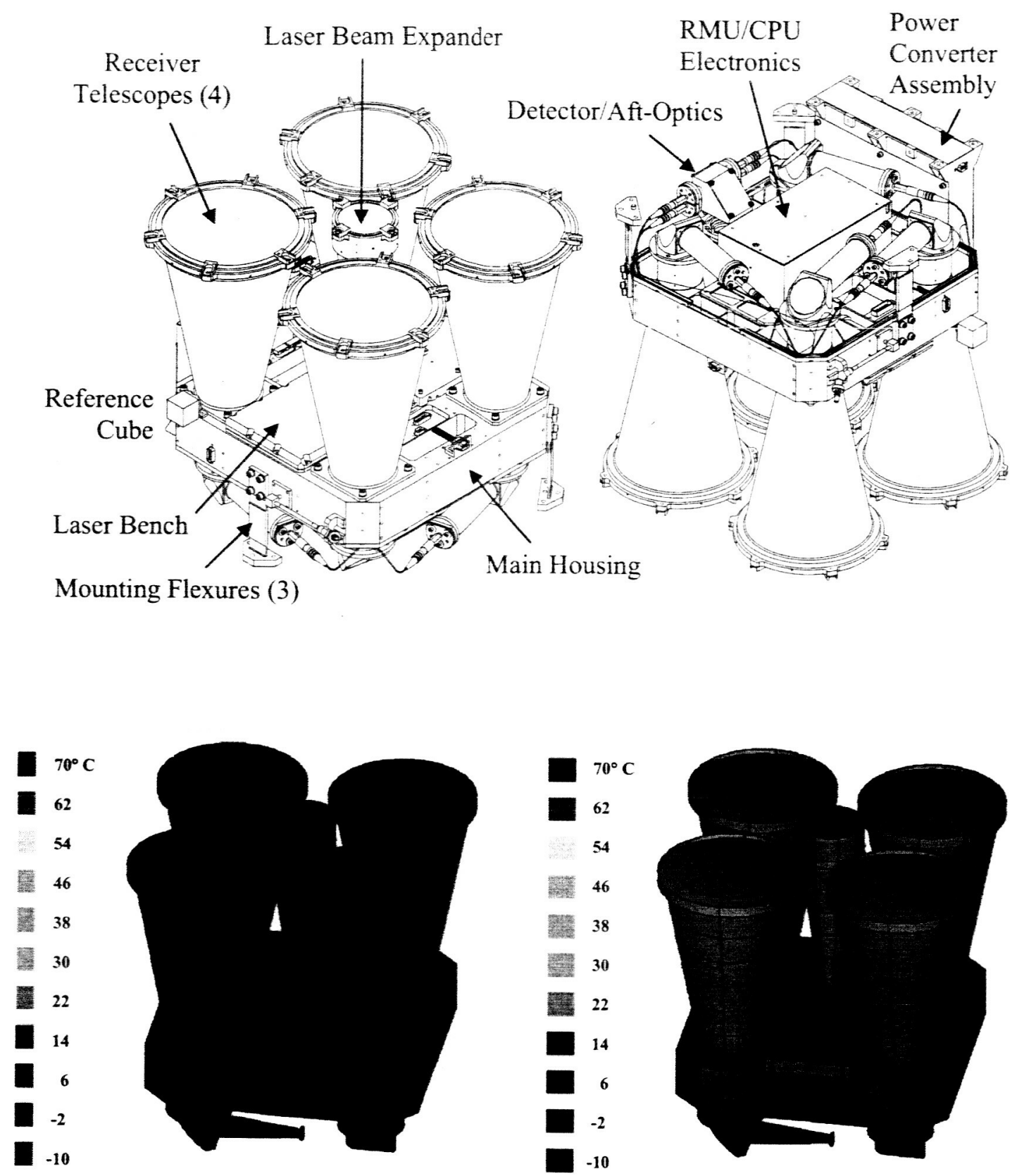


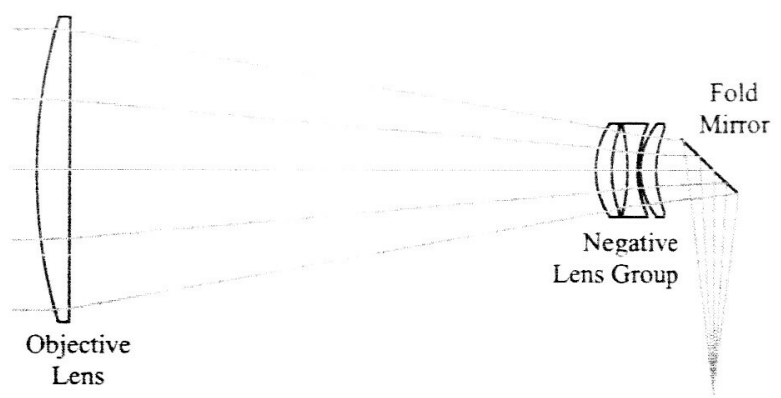

Fiber-Optic
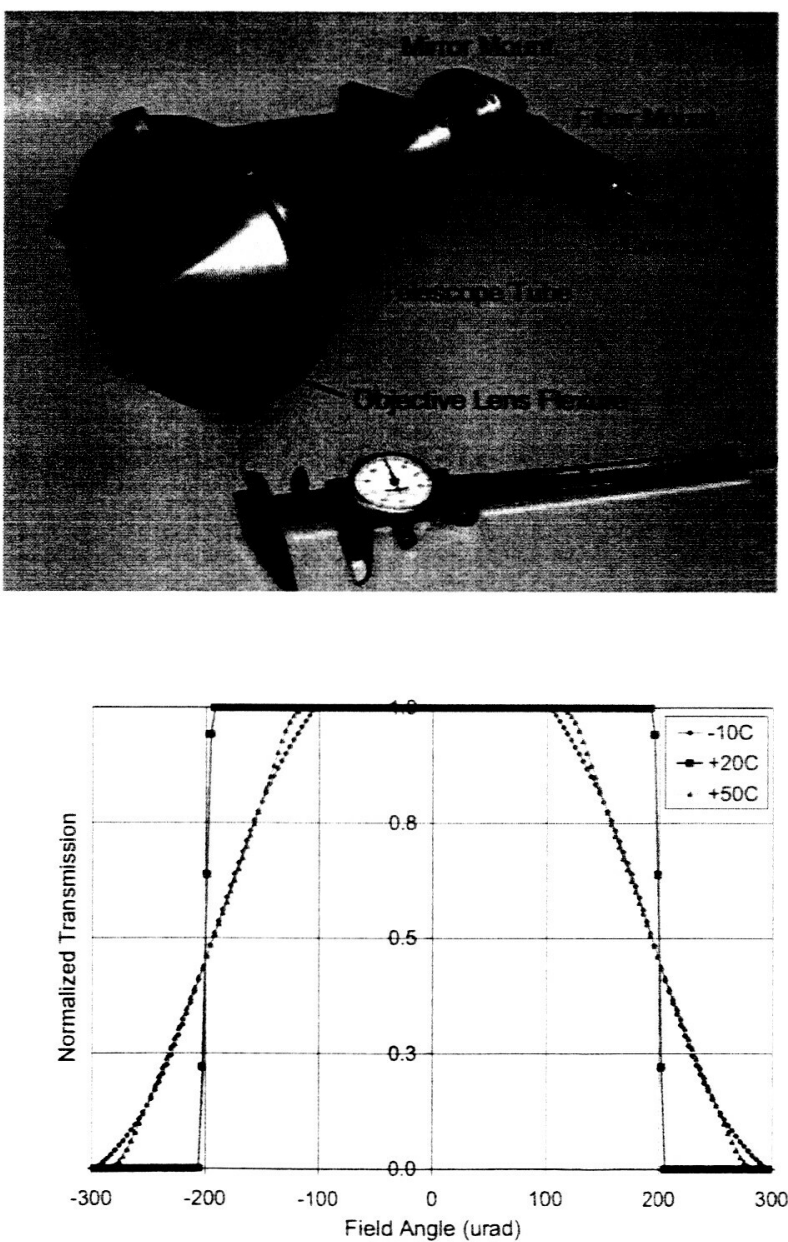

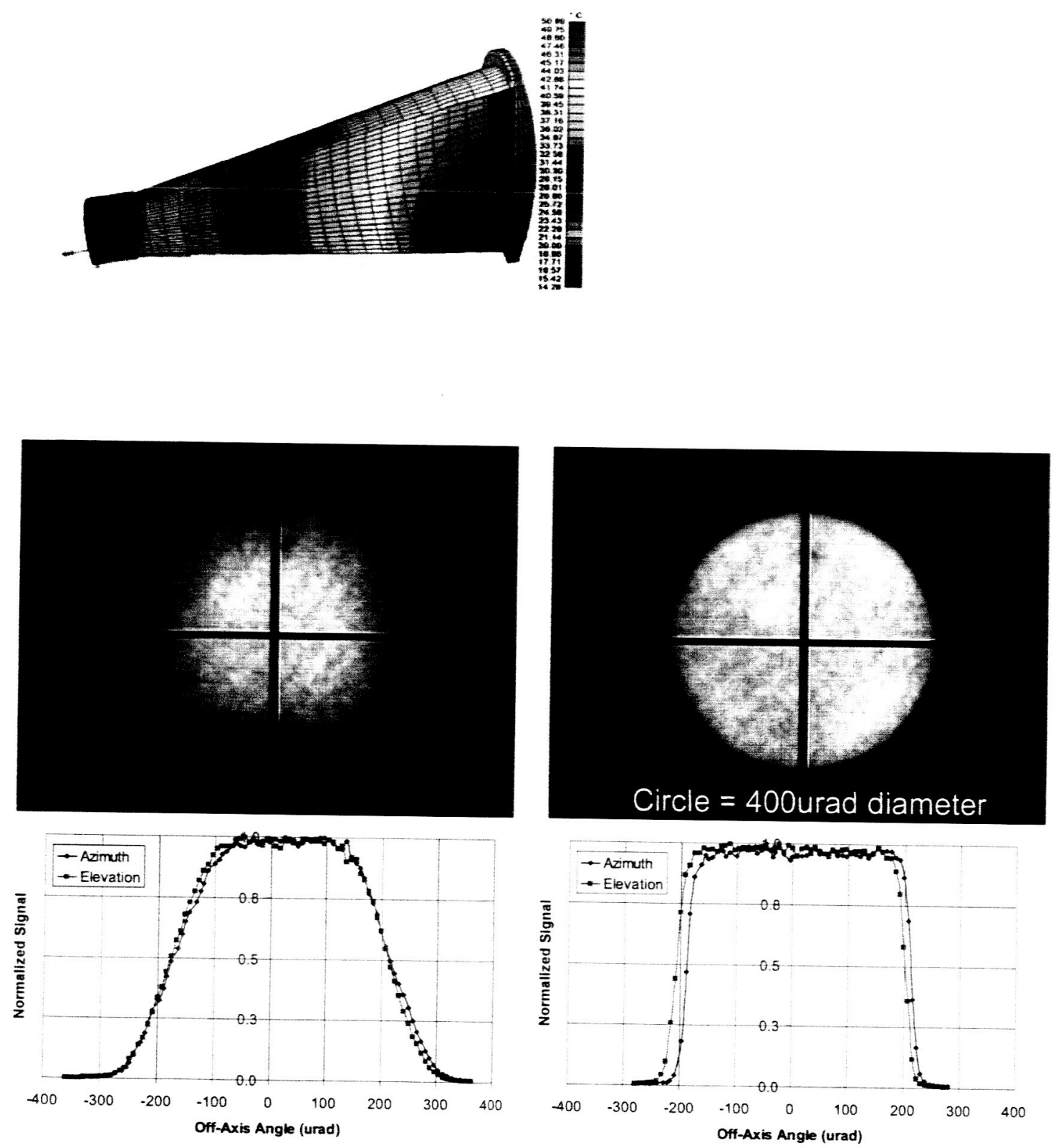

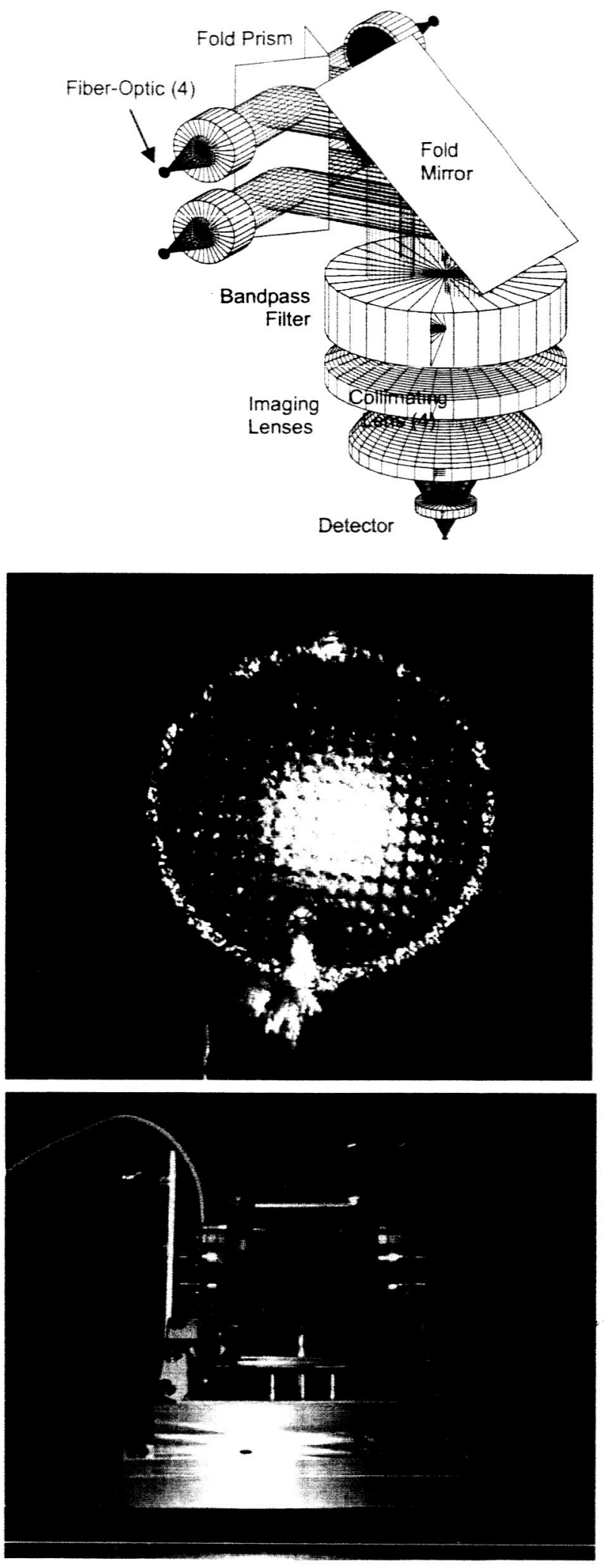

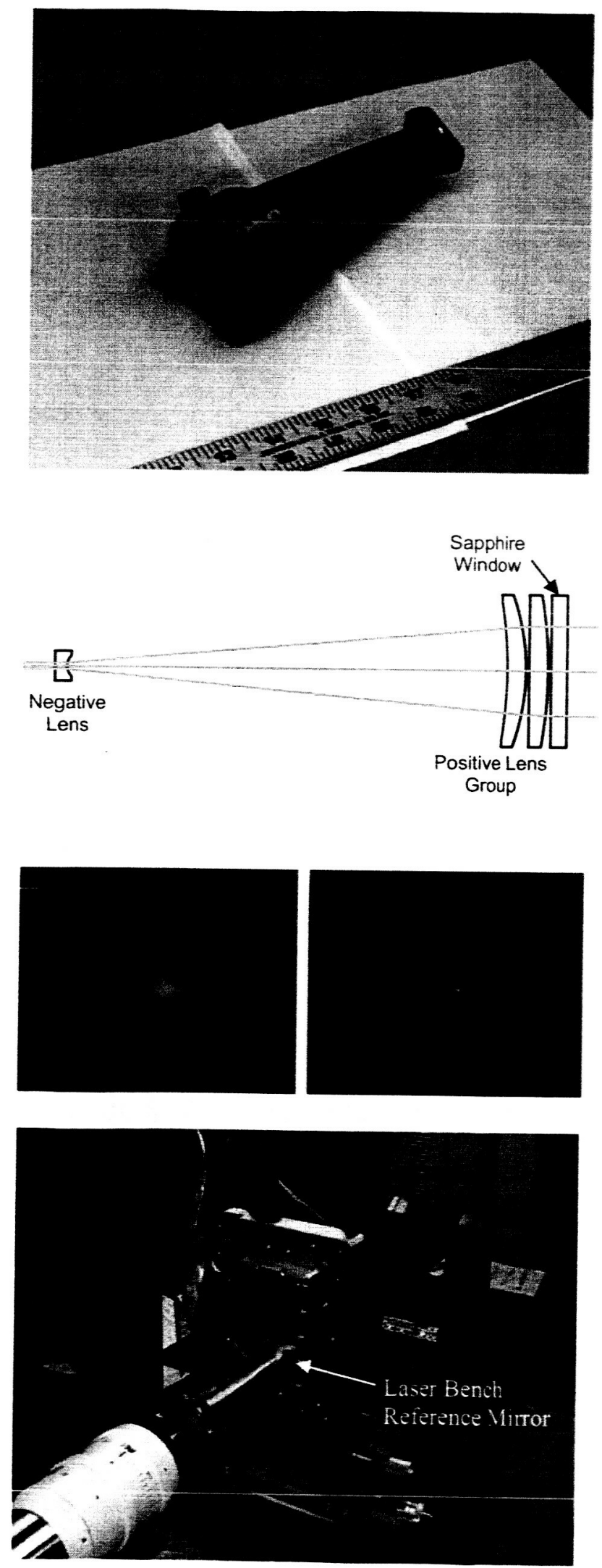

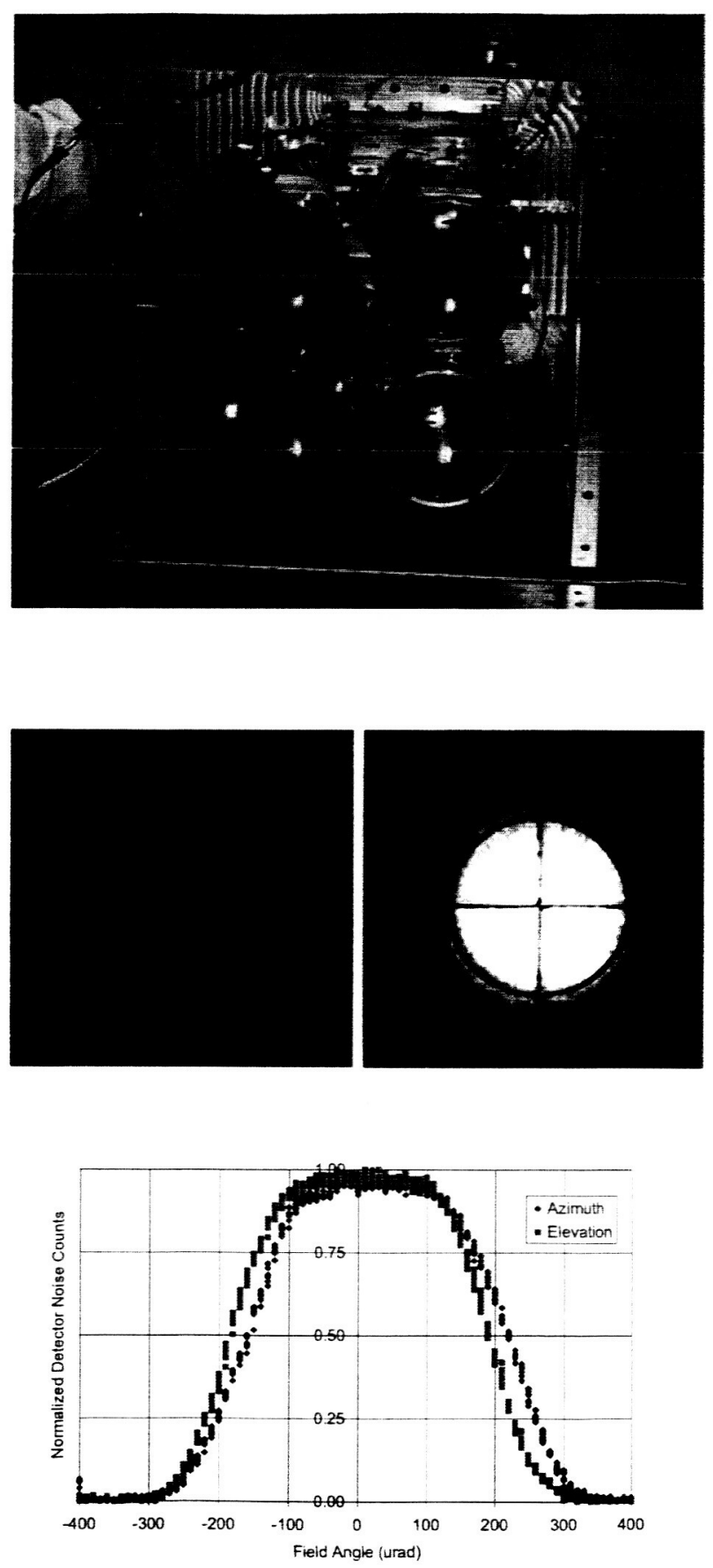


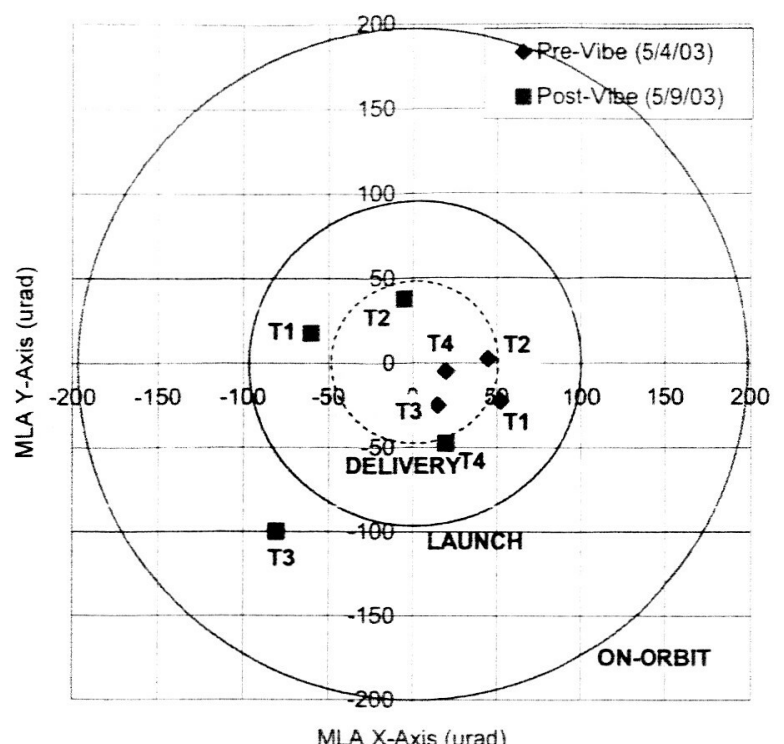

MLA X-Axis (urad)

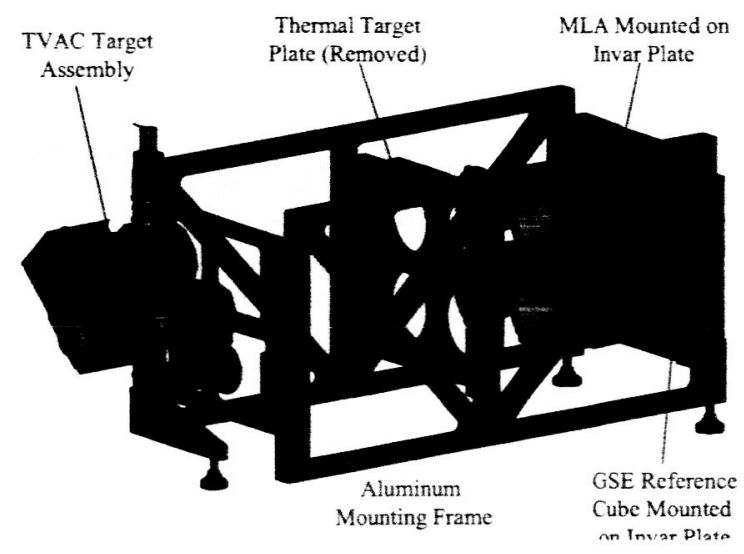



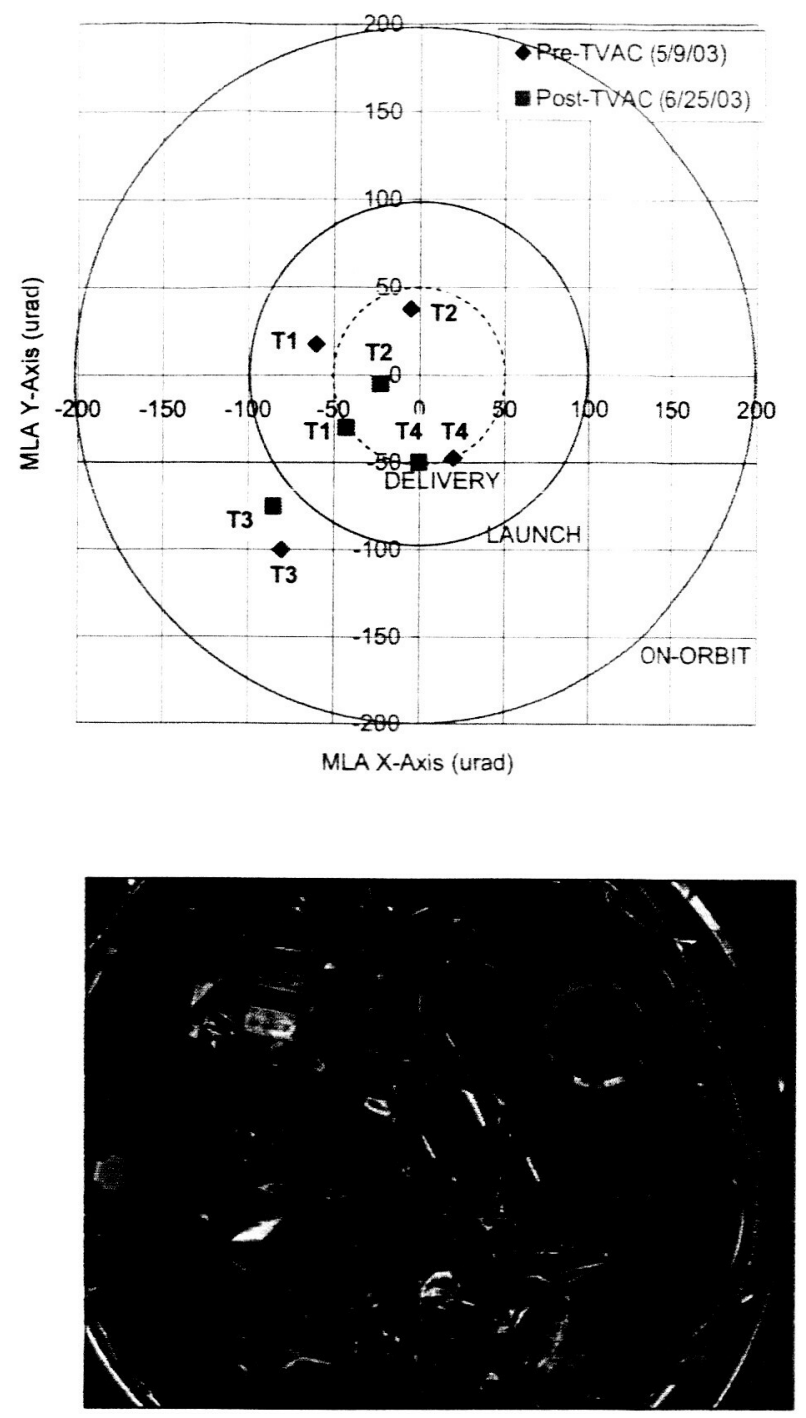


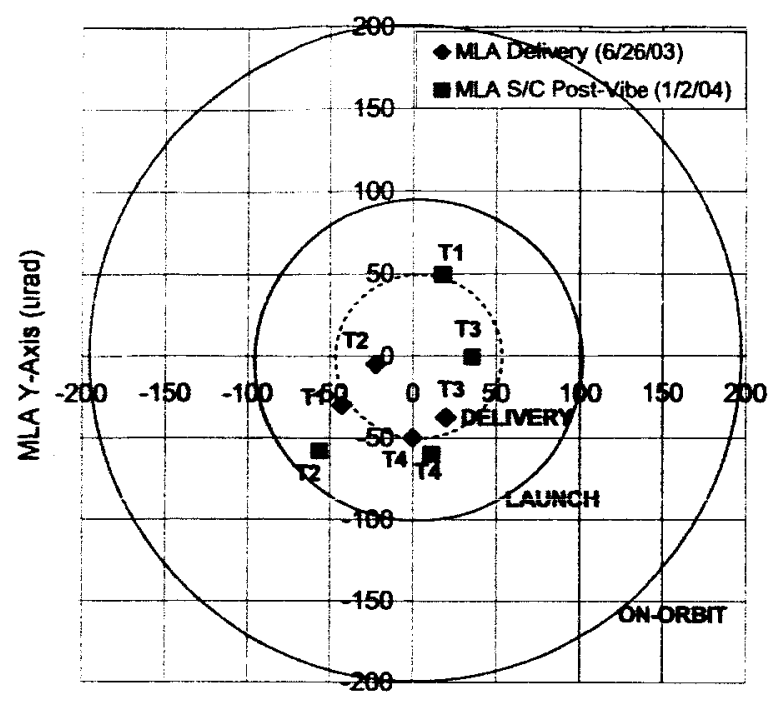

MLA X-Axis (urad)

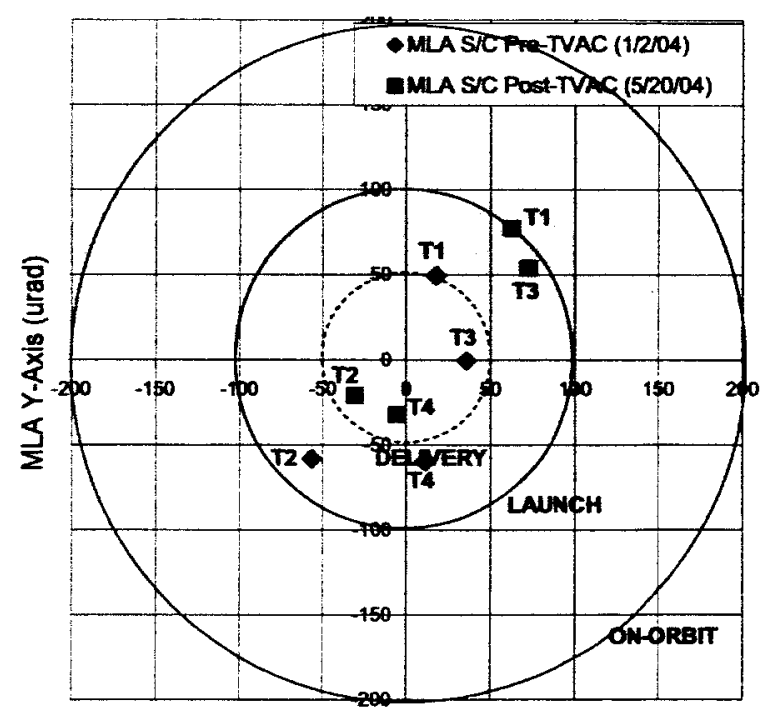

MLAX-Axis (urad) 
Table Captions:

1. MLA Top-Level Optical Specifications

2. MLA Optical Alignment Requirements

3. MLA Optical Assemblies Thermal Qualification Summary

\begin{tabular}{|c|c|c|c|}
\hline \multicolumn{2}{|c|}{ Transmitter } & \multicolumn{2}{|c|}{ Receiver } \\
\hline Wavelength & $1064 \mathrm{~nm}$ & Aperture & $417 \mathrm{~cm}^{2}$ \\
\hline Pulse Energy & $20 \mathrm{~mJ}$ & FOV (dia.) & $400 \mu \mathrm{rad}$ \\
\hline Pulse Width & $6 \mathrm{~ns}$ & Filter (FWHM) & $0.7 \mathrm{~nm},>80 \% \mathrm{~T}$ \\
\hline Repetition Rate & $8 \mathrm{~Hz}$ & Detector (dia.) & $0.7 \mathrm{~mm}$ SiAPD \\
\hline Divergence (1/e & $80 \mu \mathrm{rad}, \mathrm{TEM} 00$ & Stray Light & off-axis $<$ on-axis \\
\hline
\end{tabular}

\begin{tabular}{|c|c|c|}
\hline \multicolumn{2}{|c|}{ A instrument htegration } & Spec \\
\hline $\begin{array}{l}1 \\
2 \\
\end{array}$ & $\begin{array}{l}\text { Laser parallel to receiver telescopes } \\
\text { Laser perpendicular to MLA mounting plane }\end{array}$ & $\begin{array}{l}2 \mathrm{mrad} \\
<5 \mathrm{mrad}\end{array}$ \\
\hline \multicolumn{2}{|r|}{ B. Instument Aligrment } & Spec \\
\hline $\begin{array}{l}1 \\
2\end{array}$ & $\begin{array}{l}\text { Receiver telescopes boresite to laser } \\
\text { Knowledge of laser pointing angle (relative to MA Reference Cube) }\end{array}$ & $\begin{array}{l}+1-50 \text { urad } \\
+-50 \text { yrad }\end{array}$ \\
\hline \multicolumn{2}{|r|}{ C. Instrument Stability } & Spec \\
\hline $\begin{array}{l}1 \\
2\end{array}$ & $\begin{array}{l}\text { Laser pointing angle (relative to MLA mounting plane) } \\
\text { Receiver telescopes boresite to laser }\end{array}$ & $\begin{array}{l}+/ 50 \mu \mathrm{rad} \\
+1-100 \mu \mathrm{rad}\end{array}$ \\
\hline
\end{tabular}

\begin{tabular}{|c|c|c|c|c|c|c|c|}
\hline & & & \multicolumn{4}{|c|}{ Thermal Test Parameters } & \\
\hline Optical Assembly & Qty & $\mathrm{P} / \mathbf{N}$ & Cycles & Range $\left({ }^{\circ} \mathrm{C}\right)$ & Rate $\left({ }^{\circ} \mathrm{C} / \mathrm{hr}\right)$ & Dwell (hr) & Next Assembly \\
\hline Receiver Telescope & 6 & 2053182 & $\begin{array}{l}4 \\
2\end{array}$ & +60 to -30 & $\begin{array}{l}+60 /-20 \\
+60 /-20\end{array}$ & 4.0 & Instrument \\
\hline Fold Mirror Sub-Assembly & 6 & 2053201 & $\underline{2}$ & +60 to -30 & $+60 /-20$ & 4.0 & Receiver Telscope \\
\hline Aft-Optics Mirror Sub-Assemblv & $\begin{array}{l}2 \\
3\end{array}$ & 2054970 & 4 & $\begin{array}{l}+40 \text { to }-30 \\
+60 \text { to }-30\end{array}$ & $\begin{array}{l}30 \\
30\end{array}$ & 4.0 & Instrument \\
\hline Prism Sub-Assembly & 3 & 2054971 & 2 & +60 to -30 & 30 & 4.0 & Aft-Optics \\
\hline Collimating Lens Sub-Assembly & 2 & 2054990 & 2 & +40 to -30 & 30 & 4.0 & Aft-Optics \\
\hline Focusing Lens Sub-Assembly & 2 & 2054798 & 2 & +40 to -30 & 30 & 4.0 & Aft-Optics \\
\hline Fiber-Optics & 3 & 2053208 & 90 & +50 to -30 & 120 & 0.25 & Instrument \\
\hline Beam Expander & 2 & 2053322 & 2 & +75 to -30 & $+601-20$ & 4.0 & Laser Transmitter \\
\hline
\end{tabular}

